\title{
Neurobiological Impact of EMDR in Cancer
}

\author{
Sara Carletto \\ San Luigi Gonzaga University Hospital, Orbassano (Turin), Italy \\ Marco Pagani \\ Institute of Cognitive Sciences and Technologies, CNR, Rome, Italy \\ Department of Nuclear Medicine, Karolinska Hospital, Stockholm, Sweden
}

\begin{abstract}
The exposure to a life-threatening disease such as cancer may constitute a traumatic experience that in some cases may lead to the development of posttraumatic stress disorder (PTSD). In recent years, several studies investigated this syndrome in patients with cancer, but few focused on the underlying neurobiology. The aim of this work was to review the current literature of neurobiology of PTSD in oncological diseases, focusing on a comparison with the results of neurobiological studies on PTSD in nononcological patients and on treatments resulted effective for such disorder. Brain structures having a role in the appearance of PTSD in psycho-oncology, and in particular, in intrusive symptoms, seem to be the same involved in non-oncologic PTSD. These findings may have important implications also at clinical level, suggesting that psychotherapies found to be effective to treat PTSD in different populations may be offered also to patients with cancer-induced posttraumatic symptoms. Further studies are needed to deepen our knowledge about cancer-related PTSD neurobiology and its treatment, aiming at transferring the results into clinical practice.
\end{abstract}

Keywords: posttraumatic stress disorder (PTSD); cancer; neurobiology; eye movement desensitization and reprocessing (EMDR); cognitive behavioral therapy (CBT); psychotherapy

$\mathbf{P}$ osttraumatic stress disorder (PTSD) symptoms seem to reflect a persistent, abnormal adaptation of neurobiological systems to the stress following experienced trauma. In the recent years, several studies have highlighted how psychological trauma can cause anatomical and functional changes in specific brain areas associated with the onset of posttraumatic symptoms. Thanks to the development of neuroimaging techniques such as magnetic resonance imaging (MRI), functional magnetic resonance imaging (fMRI), positron emission tomography (PET), and single photon emission computed tomography (SPECT) it has been possible to identify the brain regions and circuits involved in the pathophysiology of PTSD.

Amygdala, medial prefrontal cortex (mPFC), and hippocampus are those brain regions that primarily appear to be pathologically involved in PTSD (Martin, Ressler, Binder, \& Nemeroff, 2010; Shin, Rauch, \& Pitman, 2006; Wager, Lindquist, \& Kaplan, 2007; Yehuda \& LeDoux, 2007).
According to the neurobiological model of PTSD (Shin et al., 2006), there is evidence for an heightened responsivity of the amygdala, implying an exaggerated response to fear as well as to threat-related, ambiguous, and uncertain stimuli (Herry et al., 2007; Sander, Grafman, \& Zalla, 2003). On the other hand, there is a diminished responsivity of the regions of the prefrontal cortex (including rostral cingulate cortex and ventromedial frontal gyrus), and this has been linked to a partial failure in the appropriate functional inhibition of the activity of the amygdala by the mPFC (Etkin \& Wager, 2007). In addition, evidence of decreased hippocampal volume and neuronal integrity as well as impaired hippocampal function is associated with explicit memory impairment (Liberzon \& Sripada, 2008).

This neurobiological model is now widely accepted by the scientific community, and despite the divergent results because of the different neuroimaging techniques used to the low sample size in the most of studies and to the heterogeneity of 
patients' symptoms, it has been validated in research and clinical settings.

Decades of neuroimaging studies also showed other regions involved in PTSD. Insula hyperactivity is associated with trauma reliving, intrusions, and avoidant behaviors (Nardo et al., 2011). Deactivation of the Broca's area may explain patients' difficulty in describing and cognitively restructuring their traumatic experience (Hull, 2002).

A significant activation of retrosplenial cortex and precuneus may account for reexperiencing the precipitating event in the form of recurrent, distressing images and recollections (Sartory et al., 2013). Thalamus is deactivated in patients with PTSD (Lanius et al., 2004), and the alterations in thalamocortical connectivity may be implicated in excessive fear recall, failure of expression and maintenance of extinction memory, and heightened traumatic remembrance (Yin et al., 2011). Cerebellum volume is smaller in patients with PTSD, and this reduction is associated with the magnitude of the PTSD symptoms (Baldaçara et al., 2011). On the other hand, right caudate volume was found to increase in PTSD, possibly because of selective lateralized activation of the corticostriatal circuits subserving processing of trauma resulting in neuroplastic changes (Looi et al., 2009).

Also, anterior cingulate cortex (ACC) was found to be implicated in the pathophysiology of PTSD. Corrigan (2002) underlined that ACC is divided in two parts: an affective subdivision in the ventral ACC and a cognitive subdivision in the dorsal ACC that reciprocally inhibit each other. In PTSD, this reciprocal inhibition is unbalanced in favor of a greater activation of the ventral affective ACC versus a deactivation of the cognitive dorsal ACC. This hypothesis was recently supported by a neuroimaging study (Bing et al., 2013) that found focal thinning in the dorsal ACC in patients with PTSD.

Finally, some studies (Lanius, Brand, Vermetten, Frewen, \& Spiegel, 2012; Lanius et al., 2010; Nardo et al., 2013) have highlighted an important distinction between two different types of posttraumatic symptoms. The first is the so-called "hyperaroused" one, characterized by emotion dysregulation that involves emotional undermodulation, mediated by the failure of prefrontal inhibition on hyperactivity in limbic regions resulting in an increased activation of the alarm systems. The other is the so-called "dissociative" one, characterized by an emotion dysregulation that involving emotional overmodulation mediated by midline prefrontal inhibition of the limbic regions.
In summary, these studies have underscored the existence of specific neural models connected to different posttraumatic clinical presentations, involving several cerebral regions.

\section{Neurobiological Correlates of Posttraumatic Stress Disorder in Oncological Diseases}

The diagnosis and treatment of a life-threatening disease such as cancer involves exposure to psychological and physical stress and may constitute a traumatic experience for most people. In recent years, several studies investigated the characteristics of cancer-related PTSD (Abbey, Thompson, Hickish, $\&$ Heathcote, 2015; Andrykowski \& Kangas, 2010; Kangas, Henry, \& Bryant, 2002). These investigations converge in identifying risk factors to developing PTSD, such as the severity of cancer, the recent diagnosis, and the presence of treatment side effects and pain. Furthermore, individual factors such as previous trauma and poor social support may also play a role in PTSD development.

Several studies have shown that $5 \%-35 \%$ of patients with cancer might suffer from posttraumatic symptoms related to their disease (Kangas et al., 2002; National Cancer Institute, 2015). These symptoms are often displayed as constant worries, fears of recurrence, nightmares or flashbacks about the illness or treatments, and a sense of shortened future. Patients with cancer often show avoidant behavior, focused on places, events, and people connected to their cancer experience. They also may experience irritability, difficulty sleeping, restlessness, and fatigue.

Intrusive symptoms are the preeminent ones (Matsuoka, Nagamine, \& Uchitomi, 2006) and have a prevalence varying from $11 \%-45 \%$ according to different studies.

In the last decade, neuroimaging techniques, initially used to diagnose tumors and to detect changes in brain caused by the presence of oncological disease, have also had a strong role in delineating psychological and psychiatric disorders linked to cancer diseases (Chiaravalloti et al., 2013; Tashiro, 2004).

Therefore, these studies have been useful in highlighting the neurobiological correlates of PTSD symptoms in patients with cancer; the main results of which will be described in this following section.

\section{Hippocampus}

Most of the structural studies converge in describing a reduction in hippocampal volume in patients with PTSD (for a review, see Shin et al., 2006). Some recent volumetric analyses have explored the 
presence of this association even in patients with oncological pathology.

Nakano and colleagues (2002) showed a significant reduction in left hippocampal volume associated with intrusive memories in a group of women with breast cancer. A reduction of hippocampal volume was also seen in patients with major depression without cancer (Bremner et al., 2000; Sheline, Wang, Gado, Csernansky, \& Vannier, 1996), but this result was not confirmed in women with a major depressive episode following breast cancer diagnosis (Inagaki et al., 2004). This discrepancy may be mainly because of two factors: (a) the length of depressive episodes, which was relatively shorter in patients with breast cancer, and (b) gender, because a reduction of hippocampal volume was more prevalent in men suffering from depression rather than in women (Frodl et al., 2002).

Moreover, Hara et al. (2008) did not find any association between hippocampal volume and PTSD, but they highlighted an inverse correlation between intrusive symptoms and hippocampal volume, consistent with the results of Nakano et al. (2002). As suggested by the latter investigation, this could mean that intrusive symptoms, and not PTSD in general, may be associated with a smaller hippocampus.

Another study (Bergouignan et al., 2011) found that a reduction in hippocampal volume was associated with the diagnosis of cancer per se, regardless the presence of PTSD or major depression. "Cancer experience" by itself appears to be associated with a reduction in the hippocampal volume in accordance with Nakano et al. (2002).

The etiology of decreased hippocampal volume in PTSD is not yet established, but two major hypotheses have been proposed. The first one proposes that trauma exposure or posttraumatic response may affect hippocampal volume because of the neurotoxicity caused by cortisol (Bremner et al., 2000). The second assumption suggests that a smaller hippocampus may be a vulnerability factor to developing PTSD after exposure to a stressful event (Gilbertson et al., 2002; Gurvits et al., 2006). In general, a hippocampal deficit was seen in patients whose traumatic experiences were supposedly more intense and prolonged.

Moreover, it is important to point out that current therapies for cancer may represent a confounding factor. Several studies have shown that chemotherapy and radiotherapy can cause deleterious cell loss and may affect adult hippocampal neurogenesis, contributing to memory deficits as well as psychiatric symptoms, such as depression (Pereira Dias et al., 2014). A temporal pattern of chemotherapy toxicity on brain regions has not been described yet, neither there is knowledge about possible longlasting effects of chemotherapy and their impact on the development and treatment of PTSD. More work is needed.

\section{Amygdala}

Studies focusing on reduced amygdala volume in patients with cancer found divergent results. Matsuoka, Yamawaki, Inagaki, Akechi, and Uchitomi (2003) showed a significant reduction in the volume of amygdala associated with intrusive memories. A similar result was found also in patients with cancer and symptoms of depression (Yoshikawa et al., 2006). On the other hand, Hara et al. (2008) did not replicate this finding in patients with PTSD or intrusive symptoms related to cancer. In these studies, the different duration and severity of PTSD necessary to cause volumetric changes of the amygdala may account for the divergent results.

Moreover, recent studies on imaging gene $\mathrm{x}$ environment interaction (Bogdan, Pagliaccio, Baranger, \& Hariri, 2016; Pagliaccio et al., 2014) suggest that genetic variation within the neuroendocrine stress hormone system and early environmental factors contribute to variation in stress reactivity and limbic brain volumes (i.e., amygdala and hippocampus), which, in turn, might result as a risk factor to develop psychopathology.

\section{Orbitofrontal Cortex}

To date, only one study (Hakamata et al., 2007) has investigated the role of orbitofrontal cortex (OFC) in patients with posttraumatic symptoms linked to cancer experience. The results showed that gray matter volume in OFC of women with PTSD was significantly lower than in women without PTSD. However, to evaluate the effect of chemotherapy on brain, a PET study recruiting patients with Hodgkin's lymphoma was recently conducted (Chiaravalloti et al., 2013). Hypometabolism in frontal cortex-in particular in the PFC, in the OFC, and ACC-after 2 months of chemotherapy was found. These changes disappeared about six months later, at the end of chemotherapy, upon symptoms disappearance. Although in this study an assessment of psychological symptoms was not carried out, it is worth noting that brain areas found to be hypometabolic after 2 months of chemotherapy were the same as those implicated in affective disorders involving the limbic circuitry and alterations in PFC and ACC suggests the presence of PTSD or depressive state in the acute phase. 


\section{Summary of Results}

The earlier described studies highlight how intrusive symptoms in oncology patients can be considered as the core of PTSD also at a neurobiological level.

These recurrent and distressing memories related to the disease play a significant role in patients' functioning, and they may adversely affect the acceptance of the disease (Whitaker, Brewin, \& Watson, 2008) leading to symptoms of depression (Brewin, Watson, McCarthy, Hyman, \& Dayson, 1998).

Moreover, these findings highlight that brain structures having a role in the appearance of posttraumatic symptoms in psycho-oncology, and in particular intrusive symptoms, seem to be the same implicated in non-oncologic PTSD.

These investigations have some limitations that have to be acknowledged. Almost all neuroimaging studies conducted in psycho-oncology focused on women with breast cancer, possibly because of the higher prevalence of this type of cancer compared to other tumors. Different populations of patients with cancer have to be taken into account to evaluate specific characteristics for any kind of disease as well as the effects of chemotherapy on different brain regions and their possible mediating/confounding effect on PTS symptoms. Moreover, recent gene $\mathrm{x}$ environment interaction research may contribute to widen the understanding of the different etiological factors of PTSD.

\section{Neurobiological Correlates of Psychotherapy Treatment}

In the recent years, several studies using different methodologies, such as MRI, fMRI, PET, SPECT, and electroencephalography (EEG) have investigated the effectiveness of psychotherapy in PTSD, with a specific focus on its neurobiological substrate and trying to reveal the neural changes associated with treatment.

As reported earlier, brain structures possibly responsible for posttraumatic symptoms are amygdala and hippocampus (hyperaroused) as well as PFC (deactivated). Furthermore, the deactivation of Broca's area may explain the difficulty, for people suffering from this disorder, in accessing a semantic knowledge of their emotional experiences. This element can therefore question the effectiveness of some "talking therapies" (Hull, 2002) and may instead suggest the use of psychological treatments allowing a multimodal and multisensory access to traumatic memories.
To date, trauma-focused cognitive behavioral therapy, eye movement desensitization and reprocessing (EMDR) therapy, and stress management are those treatments recognized as more effective in reducing PTS symptoms (Bradley, Greene, Russ, Dutra, \& Westen, 2005) and considered as elective treatments for PTSD by numerous international guidelines (American Psychiatric Association, 2006; Bisson \& Andrew, 2007; National Collaborating Centre for Mental Health, 2005; Tol, Barbui, \& van Ommeren, 2013).

Most of the neuroimaging studies have focused on EMDR therapy, whereas only a few have investigated the neurobiological effects of therapies such as cognitive behavioral therapy (CBT) and brief eclectic psychotherapy (Pagani \& Cavallo, 2014; Pagani, Högberg, Fernandez, \& Siracusano, 2013).

EMDR therapy is an information-processing therapy that focuses on trauma elaboration. A distinct characteristic of EMDR therapy is the use of alternating bilateral stimulation (eye movements, tactile, or audio) that appears to produce a physiological effect promoting accelerated reprocessing of dysfunctionally stored information related to the traumatic event.

Studies that have used SPECT to compare brain activation before and after EMDR therapy showed that significant changes in blood flow occurred mainly in limbic areas and in PFC. This normalization was also associated with symptoms remission resulting in a reduction of flashbacks, intrusive memories, and of the feeling of reliving the trauma even at a somatic level (Lansing, Amen, Hanks, \& Rudy, 2005; Oh \& Choi, 2007; Pagani et al., 2007).

Other MRI and fMRI studies have investigated hippocampal volume in patients treated with EMDR (Bossini et al., 2012) showing an increased hippocampal volume in association with improvement of posttraumatic symptoms.

Nardo and colleagues (2010) also showed with MRI that patients who had not responded to EMDR therapy showed, before the therapy sessions, a decrease of gray matter density in various limbic regions (posterior cingulate, parahippocampal cortex, and insular cortex) that may prevent access to target regions, rendering EMDR ineffective.

EMDR therapy was also evaluated using EEG (Harper, Rasolkhani-Kalhorn, \& Drozd, 2009; Lamprecht et al., 2004; Propper and Christman, 2008; Propper, Pierce, Geisler, Christman, \& Bellorado, 2007). Lamprecht and collaborators (2004) investigating evoked potentials related to the traumatic event before and after an EMDR therapy session suggested that clinical improvements may be linked to changes 
in information processing associated with a reduction of new stimuli orientation and with lower levels of arousal after the treatment. Propper and colleagues (Propper \& Christman, 2008; Propper et al., 2007) examined the effects of eye movements on interhemispheric coherence and found them to facilitate episodic memory and to result in a decreased interhemispheric coherence in anterior PFC. This might lead to consolidation of the traumatic memory of the event, allowing a reduction of intrusive symptoms. Furthermore, the brain state during the bilateral stimulation phase of EMDR therapy was found to be similar to that during slow-wave sleep (Harper et al., 2009), the condition in which new information is edited and stored in semantic memory (Stickgold, 2002; Stickgold, Hobson, Fosse, \& Fosse, 2001).

Although different neurobiological mechanisms underlying the efficacy of EMDR therapy and bilateral stimulation have been described (for review, see Bergmann, 2010, and McGuire, Lee, \& Drummond, 2014), no definitive consensus has been reached yet.

The effectiveness of CBT was investigated by Rabe, Zoellner, Beauducel, Maercker, and Karl (2008) revealing by EEG a decrease of anterior right activation associated with a reduction of posttraumatic symptoms and showing that CBT treatment may be accompanied by adaptive changes in brain function.

CBT was also investigated using MRI. Bryant, Felmingham, Kemp, et al. (2008) found a greater rostral ACC volume, also correlated with symptoms reduction, in those patients who reported an improvement of posttraumatic symptoms in comparison with those resistant to the treatment.

The same researchers using fMRI showed also that poor symptoms improvement after CBT was associated with a pretreatment observation of greater amygdala and ventral ACC activations, inferring that an exaggerated response to fear stimuli can be a key factor in limiting psychotherapeutic treatment effectiveness (Bryant, Felmingham, Whitford, et al., 2008).

The efficacy of an exposure-based treatment for patients with PTSD was assessed in a SPECT study (Peres et al., 2007). An increased activity after treatment was observed in parietal lobes, in the left hippocampus, in the thalamus, and in the left PFC, suggesting that psychotherapy could influence the development of narrative memories, overlaying the neural substrates of previous traumatic memories. Another study (Felmingham et al., 2007) investigated by fMRI the efficacy of CBT for PTSD reporting that changes in PTSD severity correlated with changes in ACC activation and inversely correlated with right amygdala activation. These changes are associated with a better ability to result in extinction of the fear response.

Lindauer and colleagues (Lindauer et al., 2008; Lindauer et al., 2005) assessed the neurobiological and clinical outcome of treatment with brief eclectic psychotherapy. In the MRI study (Lindauer et al., 2005), they showed no changes in hippocampal volume after treatment even in patients who reported symptoms improvement. On the other hand, in their second study (Lindauer et al., 2008) performed by SPECT, they highlighted changes in dorsolateral PFC associated with a treatment positive outcome.

Recently, a ground-breaking study investigated, with an accurate time resolution, the real-time brain changes occurring during a therapy session, overcoming previous static observations before and after the treatment. Pagani and colleagues (Pagani et al., 2011; Pagani et al., 2012) compared the EEG of the first (in which patients were symptomatic) and of the last (in which patients were asymptomatic) EMDR therapy sessions. A greater temporo-occipital cortex activation was observed during the latter, primarily on the left side. The limbic activation prevailing during typical EMDR alternate bilateral stimulation that was present in OFC, in PFC, and in ACC during the first EMDR session moved to associative regions after EMDR treatment. These findings, confirmed recently in a much larger patient and control subject cohorts (Pagani et al., 2015), pointed out that traumatic memories move from an implicit subcortical state to an explicit cortical state, where different brain regions contribute to process and re-elaborate the experience. A normalization of activation after trauma processing was highlighted, which can be interpreted as the neurobiological correlates of symptoms improvement.

\section{Summary of Results}

Overall, the results of these studies indicate a posttreatment reversal of the prefrontal and limbic abnormalities, which were clearly recognized at the pretreatment stage and are frequent neuroimaging findings in patients with PTSD. The PFC recovers its inhibitory role, reducing amygdala hyperactivation and the corresponding cortical hyperarousal. Also, hippocampus seems to regain its function, promoting a contextualization and a consolidation in a declarative form of the memories related to the traumatic event.

These findings are also consistent with long-lasting clinical improvements (Högberg et al., 2008), demonstrating that psychotherapy efficacy is strongly related to the normalization of brain functions. In particular, EMDR therapy appears to promote a shift of the 
traumatic material from an implicit subcortical to an explicit cortical status where different brain regions participate in processing the experience.

Patients who do not improve after EMDR therapy or CBT show a decrease of gray matter density in posterior cingulate, parahippocampal cortex, and insular cortex and increased activation of the amygdala and ventral ACC. These regions are well known to be implicated in processes such as the integration of cognitive and affective processes, encoding and retrieval of autobiographical and episodic memories, emotional processing, interoceptive awareness, self-referential conscious experience, and extinction of fear. Deficits in these areas can constitute a key factor in limiting the efficacy of psychotherapeutic treatments.

\section{Implications for Cancer-Related PTSD Treatment}

Although all studies assessing the prevalence of PTSD in patients with cancer recommend interventions for this disorder (Abbey et al., 2015; Andrykowski \& Kangas, 2010; Kangas et al., 2002), little research has been carried out on the psychological intervention that may reduce PTSD symptoms in this population. To date, there is only one study that examined the efficacy of EMDR therapy and CBT in oncology patients with PTSD (Capezzani et al., 2013). Moreover, no neuroimaging studies have evaluated the efficacy of psychotherapy for posttraumatic symptoms in patients with cancer.

Intrusive symptoms appear to be the core of PTSD in oncology patients both at a clinical and neurobiological level. Brewin et al. (1998) showed that such symptoms are also present in patients with cancer and depression and suggest that these memories correspond to events that have not received sufficient emotional processing. Therefore, these disabling signs may be present also in those patients with cancer who do not meet full criteria for a diagnosis of PTSD (i.e., subthreshold PTSD) and suffer from depression or adjustment disorder.

Our review provides a neurobiologically based support for what was suggested by the National Cancer Institute (2015), which recommends that patients with cancer receive the same therapies used to treat PTSD related to other forms of trauma.

Consideration of these recommendations may encourage future studies investigating the neurobiological impact of psychotherapies in oncology. EMDR therapy which functional effects have been demonstrated both during therapy sessions and in posttreatment assessments is an ideal candidate. Its proven ability to normalize the dysfunction of limbic areas involved in PTSD and depression might relieve patients from the psychological burden associated with oncological diseases. The only clinical study conducted so far (Capezzani et al., 2013) showed that EMDR therapy was more effective than CBT in the treatment of cancer-related PTSD, but larger samples and the application of neuroimaging techniques are necessary to deepen our understanding of the differences between these treatments.

Investigations into the role of dissociation are also needed. As reported in the first part of this work, some studies have revealed a dissociative subtype of PTSD, presenting a peculiar neurobiological pattern. They also highlighted important implications for the treatment that should be offered to patients with dissociative PTSD (Lanius et al., 2012). A recent study underscored the presence of a higher dissociation score in patients with cancer, receiving chemotherapy compared with patients who did not (Civilotti et al., 2015). These considerations emphasize why it is even more important to investigate dissociative symptoms and to clarify the role of antitumor therapies in the pathophysiology of the symptoms of cancerrelated PTSD.

This literature review on the neurobiological aspects of cancer-related PTSD suggests that the brain regions involved in PTSD in patients with cancer appear to be the same as those highlighted in studies on different psychological traumas such as road accidents, combat trauma, sexual abuse, and other stressful events considered as "classic" traumas in PTSD nosology.

It is therefore reasonable to infer that therapies, found to be effective in treating PTSD in populations who had suffered a "typical" psychological traumatic events, can be effective even in patients suffering from posttraumatic symptoms related to their physical illness because the underlying neurobiological processes appear to be similar.

\section{References}

Abbey, G., Thompson, S. B., Hickish, T., \& Heathcote, D. (2015). A meta-analysis of prevalence rates and moderating factors for cancer-related post-traumatic stress disorder. Psycho-Oncology, 24, 371-381.

American Psychiatric Association. (2006). Practice guidelines for the treatment of psychiatric disorders: Compendium 2006. Arlington, VA: Author.

Andrykowski, M. A., \& Kangas, M. (2010). Posttraumatic stress disorder associated with cancer diagnosis and treatment. In J. C. Holland, W. S. Breitbart, P. B. Jacobsen, M. S. Lederberg, M. J. Loscalzo, \& R. McCorklen (Eds.), 
Psycho-oncology (2nd ed., pp. 348-357). New York, NY: Oxford University Press.

Baldaçara, L., Jackowski, A. P., Schoedl, A., Pupo, M., Andreoli, S. B., Mello, M. F., . . Bressan, R. A. (2011). Reduced cerebellar left hemisphere and vermal volume in adults with PTSD from a community sample. Journal of Psychiatric Research, 45, 1627-1633.

Bergmann, U. (2010). EMDR's neurobiological mechanisms of action: A survey of 20 years of searching. Journal of EMDR Practice and Research, 4, 22-42.

Bergouignan, L., Lefranc, J. P., Chupin, M., Morel, N., Spano, J. P., \& Fossati, P. (2011). Breast cancer affects both the hippocampus volume and the episodic autobiographical memory retrieval. PloS One, 6, e25349.

Bing, X., Ming-Guo, Q., Ye, Z., Jing-Na, Z., Min, L., Han, C., ... Shao-Xiang, Z. (2013). Alterations in the cortical thickness and the amplitude of low-frequency fluctuation in patients with post-traumatic stress disorder. Brain Research, 1490, 225-232.

Bisson, J., \& Andrew, M. (2007). Psychological treatment of post-traumatic stress disorder (PTSD). Cochrane Database of Systematic Reviews, (3), CD003388.

Bogdan, R., Pagliaccio, D., Baranger, D. A., \& Hariri, A. R. (2016). Genetic moderation of stress effects on corticolimbic circuitry. Neuropsychopharmacology, 41(1), 275-296.

Bossini, L., Casolaro, I., Santarnecchi, E., Caterini, C., Koukouna, D., Fernandez, I., \& Fagiolini, A. (2012). Evaluation study of clinical and neurobiological efficacy of EMDR in patients suffering from post-traumatic stress disorder. Rivista Di Psichiatria, 47(Suppl. 2), 12-15.

Bradley, R., Greene, J., Russ, E., Dutra, L., \& Westen, D. (2005). A multidimensional meta-analysis of psychotherapy for PTSD. American Journal of Psychiatry, 162, 214-227.

Bremner, J. D., Narayan, M., Anderson, E. R., Staib, L. H., Miller, H. L., \& Charney, D. S. (2000). Hippocampal volume reduction in major depression. American Journal of Psychiatry, 157, 115-118.

Brewin, C. R., Watson, M., McCarthy, S., Hyman, P., \& Dayson, D. (1998). Intrusive memories and depression in cancer patients. Behaviour Research and Therapy, 36, 1131-1142.

Bryant, R. A., Felmingham, K., Kemp, A., Das, P., Hughes, G., Peduto, A., \& Williams, L. (2008). Amygdala and ventral anterior cingulate activation predicts treatment response to cognitive behaviour therapy for post-traumatic stress disorder. Psychological Medicine, 38, 555-561.

Bryant, R. A., Felmingham, K., Whitford, T. J., Kemp, A., Hughes, G., Peduto, A., \& Williams, L. M. (2008). Rostral anterior cingulate volume predicts treatment response to cognitive-behavioural therapy for posttraumatic stress disorder. Journal of Psychiatry \& Neuroscience, 33, 142-146.

Capezzani, L., Ostacoli, L., Cavallo, M., Carletto, S., Fernandez, I., Solomon, R., . . . Cantelmi, T. (2013).
EMDR and CBT for cancer patients: Comparative study of effects on PTSD, anxiety, and depression. Journal of EMDR Practice and Research, 7, 134-143.

Chiaravalloti, A., Pagani, M., Di Pietro, B., Danieli, R., Tavolozza, M., Travascio, L., . . . Schillaci, O. (2013). Is cerebral glucose metabolism affected by chemotherapy in patients with Hodgkin's lymphoma? Nuclear Medicine Communications, 34, 57-63.

Civilotti, C., Castelli, L., Binaschi, L., Cussino, M., Tesio, V., Di Fini, G., . . . Torta, R. (2015). Dissociative symptomatology in cancer patients. Frontiers in Psychology, 6, 1-7.

Corrigan, F. M. (2002). Mindfulness, dissociation, EMDR and the anterior cingulate cortex: A hypothesis. Contemporary Hypnosis, 19, 8-17.

Etkin, A., \& Wager, T. D. (2007). Functional neuroimaging of anxiety: A meta-analysis of emotional processing in PTSD, social anxiety disorder, and specific phobia. American Journal of Psychiatry, 164, 1476-1488.

Felmingham, K., Kemp, A., Williams, L., Das, P., Hughes, G., Peduto, A., \& Bryant, R. (2007). Changes in anterior cingulate and amygdala after cognitive behavior therapy of posttraumatic stress disorder. Psychological Science, 18, $127-129$.

Frodl, T., Meisenzahl, E. M., Zetzsche, T., Born, C., Groll, C., Jäger, M., . . Möller, H. J. (2002). Hippocampal changes in patients with a first episode of major depression. American Journal of Psychiatry, 159, 1112-1118.

Gilbertson, M. W., Shenton, M. E., Ciszewski, A., Kasai, K., Lasko, N. B., Orr, S. P., \& Pitman, R. K. (2002). Smaller hippocampal volume predicts pathologic vulnerability to psychological trauma. Nature Neuroscience, 5, 1242-1247.

Gurvits, T. V., Metzger, L. J., Lasko, N. B., Cannistraro, P. A., Tarhan, A. S., Gilbertson, M. W., . . Pitman, R. K. (2006). Subtle neurologic compromise as a vulnerability factor for combat-related posttraumatic stress disorder: Results of a twin study. Archives of General Psychiatry, 63, 571-576.

Hakamata, Y., Matsuoka, Y., Inagaki, M., Nagamine, M., Hara, E., Imoto, S., . . . Uchitomi, Y. (2007). Structure of orbitofrontal cortex and its longitudinal course in cancer-related post-traumatic stress disorder. Neuroscience Research, 59, 383-389.

Hara, E., Matsuoka, Y., Hakamata, Y., Nagamine, M., Inagaki, M., Imoto, S., . . Uchitomi, Y. (2008). Hippocampal and amygdalar volumes in breast cancer survivors with posttraumatic stress disorder. The Journal of Neuropsychiatry and Clinical Neurosciences, 20, 302-308.

Harper, M. L., Rasolkhani-Kalhorn, T., \& Drozd, J. F. (2009). On the neural basis of EMDR therapy: Insights from qEEG studies. Traumatology, 15, 81-95.

Herry, C., Bach, D. R., Esposito, F., Di Salle, F., Perrig, W. J., Scheffler, K., . . Seifritz, E. (2007). Processing of temporal unpredictability in human and animal amygdala, Journal of Neuroscience, 27, 5958-5966.

Högberg, G., Pagani, M., Sundin, O., Soares, J., AbergWistedt, A., Tärnell, B., \& Hällström, T. (2008). Treatment 
of post-traumatic stress disorder with eye movement desensitization and reprocessing: Outcome is stable in 35-month follow-up. Psychiatry Research, 159, 101-108.

Hull, A. M. (2002). Neuroimaging findings in posttraumatic stress disorder. Systematic review. British Journal of Psychiatry, 181, 102-110.

Inagaki, M., Matsuoka, Y., Sugahara, Y., Nakano, T., Akechi, T., Fujimori, M., . . U Uchitomi, Y. (2004). Hippocampal volume and first major depressive episode after cancer diagnosis in breast cancer survivors. American Journal of Psychiatry, 161, 2263-2270.

Kangas, M., Henry, J., \& Bryant, R. A. (2002). Posttraumatic stress disorder following cancer: A conceptual and empirical review. Clinical Psychology Review, 22, 499-524.

Lamprecht, F., Köhnke, C., Lempa, W., Sack, M., Matzke, M., \& Münte, T. F. (2004). Event-related potentials and EMDR treatment of post-traumatic stress disorder. Neuroscience Research, 49, 267-272.

Lanius, R. A., Brand, B., Vermetten, E., Frewen, P. A., \& Spiegel, D. (2012). The dissociative subtype of posttraumatic stress disorder: Rationale, clinical and neurobiological evidence, and implications. Depression and Anxiety, 29, 701-708.

Lanius, R. A., Vermetten, E., Loewenstein, R. J., Brand, B., Schmahl, C., Bremner, J. D., \& Spiegel, D. (2010). Emotion modulation in PTSD: Clinical and neurobiological evidence for a dissociative subtype. American Journal of Psychiatry, 167, 640-647.

Lanius, R. A., Williamson, P. C., Densmore, M., Boksman, K., Neufeld, R. W., Gati, J. S., \& Menon, R. S. (2004). The nature of traumatic memories: A 4-T FMRI functional connectivity analysis. American Journal of Psychiatry, 161, 36-44.

Lansing, K., Amen, D. G., Hanks, C., \& Rudy, L. (2005). High-resolution brain SPECT imaging and eye movement desensitization and reprocessing in police officers with PTSD. Journal of Neuropsychiatry and Clinical Neurosciences, 17, 526-532.

Liberzon, I., \& Sripada, C. S. (2008). The functional neuroanatomy of PTSD: A critical review. Progress in Brain Research, 167, 151-169.

Lindauer, R. J., Booij, J., Habraken, J. B., van Meijel, E. P., Uylings, H. B., Olff, M., . . . Gersons, B. P. (2008). Effects of psychotherapy on regional cerebral blood flow during trauma imagery in patients with post-traumatic stress disorder: A randomized clinical trial. Psychological Medicine, 38, 543-554.

Lindauer, R. J., Vlieger, E. J., Jalink, M., Olff, M., Carlier, I. V., Majoie, C. B., . . . Gersons, B. P. (2005). Effects of psychotherapy on hippocampal volume in out-patients with post-traumatic stress disorder: A MRI investigation. Psychological Medicine, 35, 1421-1431.

Looi, J. C., Mallerc, J. J., Pagani, M., Högberg, G., Lindberg, O., Liberg, B., . . Wahlund, L. O. (2009). Caudate volumes in public transportation workers exposed to trauma in the Stockholm train system. Psychiatry Research, 171, 138-143.
Martin, E. I., Ressler, K. J., Binder, E., \& Nemeroff, C. B. (2010). The neurobiology of anxiety disorders: Brain imaging, genetics, and psychoneuroendocrinology. Clinics in Laboratory Medicine, 32, 865-891.

Matsuoka, Y., Nagamine, M., \& Uchitomi, Y. (2006). Intrusion in women with breast cancer. In N. Kato, M. Kawata, \& R. K. Pitman (Eds.), PTSD: Brain mechanism and clinical implications (pp. 169-178). Tokyo, Japan: Springer-Verlag.

Matsuoka, Y., Yamawaki, S., Inagaki M., Akechi, T., \& Uchitomi, Y. (2003). A volumetric study of amygdala in cancer survivors with intrusive recollections. Biological Psychiatry, 54, 736-743.

McGuire, T. M., Lee, C. W., \& Drummond, P. D. (2014). Potential of eye movement desensitization and reprocessing therapy in the treatment of post-traumatic stress disorder. Psychology Research and Behavior Management,7, 273-283.

Nakano, T., Wenner, M., Inagaki, M., Kugaya, A., Akechi, T., Matsuoka, Y., . . . Uchitomi, Y. (2002). Relationship between distressing cancer-related recollections and hippocampal volume in cancer survivors. American Journal of Psychiatry, 159, 2087-2093.

Nardo, D., Högberg, G., Flumeri, F., Jacobsson, H., Larsson, S. A., Hällström, T., \& Pagani, M. (2011). Self-rating scales assessing subjective well-being and distress correlate with $\mathrm{rCBF}$ in PTSD-sensitive regions. Psychological Medicine, 41, 2549-2561.

Nardo, D., Högberg, G., Lanius, R. A., Jacobsson, H., Jonsson, C., Hällström, T., \& Pagani, M. (2013). Gray matter volume alterations related to trait dissociation in PTSD and traumatized controls. Acta Psychiatrica Scandinavica, 128, 222-233.

Nardo, D., Högberg, G., Looi, J. C., Larsson, S., Hällström, T., \& Pagani, M. (2010). Gray matter density in limbic and paralimbic cortices is associated with trauma load and EMDR outcome in PTSD patients. Journal of Psychiatric Research, 44, 477-485.

National Cancer Institute. (2015). Post-traumatic stress disorder. Retrieved from http: / / www.cancer.gov/ cancer topics / pdq/supportivecare/post-traumatic-stress / Patient

National Collaborating Centre for Mental Health. (2005). Post-traumatic stress disorder: The management of PTSD in primary and secondary care. London, United Kingdom: Royal College of Psychiatrists \& The British Psychological Society.

Oh, D. H., \& Choi, J. (2007). Changes in the cerebral perfusion after EMDR: A SPECT study of two cases. Journal of EMDR Practice and Research, 1, 24-30.

Pagani, M., \& Cavallo, M. (2014). Neuroimaging in PTSD-related psychotherapies. In R. Dierckx, A. Otte, E. de Vries, \& A. van Waarde (Eds.), PET and SPECT in psychiatry (pp. 397-410). Berlin, Germany: Springer-Verlag.

Pagani, M., Di Lorenzo, G., Monaco, L., Daverio, A., Giannoudas, I., La Porta, P., . . Siracusano, A. (2015). 
Neurobiological response to EMDR therapy in clients with different psychological traumas. Frontiers in Psychology, 6, 1614.

Pagani, M., Di Lorenzo, G., Verardo, A. R., Nicolais, G., Monaco, L., Lauretti, G., . . F Fernandez, I. (2011). Pretreatment, intratreatment, and posttreatment EEG imaging of EMDR: Methodology and preliminary results from a single case. Journal of EMDR Practice and Research, 5, 42-56.

Pagani, M., Di Lorenzo, G., Verardo A. R., Nicolais, G., Monaco, L., Lauretti, G., . . Siracusano, A. (2012). Neurobiological correlates of EMDR monitoring-An EEG study. PLoS One, 7, e45753.

Pagani, M., Högberg, G., Fernandez, I., \& Siracusano, A. (2013). Correlates of EMDR therapy in functional and structural neuroimaging: A critical summary of recent findings. Journal of EMDR Practice and Research, 7, 29-38.

Pagani, M., Högberg, G., Salmaso, D., Nardo, D., Sundin, O., Jonsson, C., . . . Hällström, T. (2007). Effects of EMDR psychotherapy on $99 \mathrm{mTc}$-HMPAO distribution in occupation-related post-traumatic stress disorder. Nuclear Medicine Communications, 28, 757-765.

Pagliaccio, D., Luby, J. L., Bogdan, R., Agrawal, A., Gaffrey, M. S., Belden, A. C., . . . Barch, D. M. (2014). Stresssystem genes and life stress predict cortisol levels and amygdala and hippocampal volumes in children. Neuropsychopharmacology, 39, 1245-1253.

Pereira Dias, G., Hollywood, R., Bevilaqua, M. C., da Luz, A. C., Hindges, R., Nardi, A. E., \& Thuret, S. (2014). Consequences of cancer treatments on adult hippocampal neurogenesis: Implications for cognitive function and depressive symptoms. Neuro Oncology, 16, 476-492.

Peres, J. F., Newberg, A. B., Mercante, J. P., Simão, M., Albuquerque, V. E., Peres, M. J., \& Nasello, A. G. (2007). Cerebral blood flow changes during retrieval of traumatic memories before and after psychotherapy: A SPECT study. Psychological Medicine, 37, 1481-1491.

Propper, R. E., \& Christman, S. D. (2008). Interhemispheric interaction and saccadic horizontal eye movements: Implications for episodic memory. Journal of EMDR Practice and Research, 2, 269-281.

Propper, R. E., Pierce, J., Geisler, M. W., Christman, S. D., \& Bellorado, N. (2007). Effect of bilateral eye movements on frontal interhemispheric gamma EEG coherence: Implications for EMDR therapy. Journal of Nervous and Mental Disease, 195, 785-788.

Rabe, S., Zoellner, T., Beauducel, A., Maercker, A., \& Karl, A. (2008). Changes in brain electrical activity after cognitive behavioral therapy for posttraumatic stress disorder in patients injured in motor vehicle accidents. Psychosomatic Medicine, 70, 13-19.
Sander, D., Grafman, J., \& Zalla, T. (2003). The human amygdala: An evolved system for relevance detection. Reviews in the Neurosciences, 14, 303-316.

Sartory, G., Cwik, J., Knuppertz, H., Schürholt, B., Lebens, M., Seitz, R. J., \& Schulze, R. (2013). In search of the trauma memory: A meta-analysis of functional neuroimaging studies of symptom provocation in posttraumatic stress disorder (PTSD). PLoS One, 8, e58150.

Sheline, Y. I., Wang, P. W., Gado, M. H., Csernansky, J. G., \& Vannier, M. W. (1996). Hippocampal atrophy in recurrent major depression. Proceedings of the National Academy of Sciences of the United States of America, 93, 3908-3913.

Shin, L. M., Rauch, S. L., \& Pitman, R. K. (2006). Amygdala, medial prefrontal cortex, and hippocampal function in PTSD. Annals of the New York Academy of Sciences, 1071, 67-79.

Stickgold, R. (2002). EMDR: A putative neurobiological mechanism of action. Journal of Clinical Psychology, 58, 61-75.

Stickgold, R., Hobson, J. A., Fosse, R., \& Fosse, M. (2001). Sleep, learning, and dreams: Off-line memory reprocessing. Science, 294, 1052-1057.

Tashiro, M. (2004). Impacts of neuroimaging on psychooncology. Psycho-Oncology, 13, 486-489.

Tol, W. A., Barbui, C., \& van Ommeren, M. (2013). Management of acute stress, PTSD, and bereavement: WHO recommendations. JAMA, 310, 477-478.

Wager, T. D., Lindquist, M., \& Kaplan, L. (2007). Metaanalysis of functional neuroimaging data: Current and future directions. Social Cognitive and Affective Neuroscience, 2, 150-158.

Whitaker, K. L., Brewin, C. R., \& Watson, M. (2008). Intrusive cognitions and anxiety in cancer patients. Journal of Psychosomatic Research, 64, 509-517.

Yehuda, R., \& LeDoux, J. (2007). Response variation following trauma: A translational neuroscience approach to understanding PTSD. Neuron, 56, 19-32.

Yin, Y., Jin, C., Hu, X., Duan, L., Li, Z., Song, M., . . Li, L. (2011). Altered resting-state functional connectivity of thalamus in earthquake-induced posttraumatic stress disorder: A functional magnetic resonance imaging study. Brain Research, 411, 98-107.

Yoshikawa, E., Matsuoka, Y., Yamasue, H., Inagaki, M., Nakano, T., Akechi, T., . . U Uchitomi, Y. (2006). Prefrontal cortex and amygdala volume in first minor or major depressive episode after cancer diagnosis. Biological Psychiatry, 59, 707-712.

Correspondence regarding this article should be directed to Sara Carletto, San Luigi Gonzaga University Hospital, University of Turin, Regione Gonzole 10, 10043 Orbassano, Turin, Italy. E-mail: sara.carletto@unito.it; saracarletto@ hotmail.com 